\title{
Processing of auditory information by the blind in spatial localization tasks
}

\author{
M. C. WANET and C. VERAART \\ University of Louvain, Brussels, Belgium
}

\begin{abstract}
Spatial localization of sonorous targets in the near space, by blindfolded sighted subjects and by early- or late-blind subjects, was investigated using a two-dimensional coordinate system (direction and distance) in various experimental conditions: The head was free or restrained, and the subject's response was either a finger pointing on a coordinate grid in open loop or a verbal assessment based on a simple reference system. The results for each group of subjects were processed by dealing separately with distance and direction parameters. They show that with early blinds, only the distance evaluation was particularly impaired. Performance did not seem to depend much on whether the head was restrained or free. Finally, direction was best evaluated by motor responses, whereas distance was more accurately judged verbally.
\end{abstract}

Besides vision, the human auditory channel contributes widely to the acquisition of information about the remote external environment. Such information plays a part in the identification of familiar objects as well as in spatial localization.

Studies focusing on the contribution of audition in spatial representation raise questions about the intersensory unity of this representation. Most approaches to spatial representation usually involve vision (for a review, see Westheimer, 1984). Other works relate to auditory spatial perception (for a review, see Masterson \& Imig, 1984). Nevertheless, a few studies have considered connections between audition and vision in the buildup of spatial representation (e.g., Warren \& Pick, 1970), and, fairly often, these studies relate to the blind's spatial perception (Millar, 1981; Rieser, Guth, \& Hill, 1982). For blind people, indeed, audition remains the main channel for providing distal information; as an example, even silent objects can be detected by blind people thanks to auditory cues, this ability constituting the so-called "obstacle sense" (Kellogg, 1962). Nevertheless, when compared with vision, auditory performance in spatial localization reaches a lower level of accuracy, as pointed out by Foulke (1982). Among the tools used to objectivate performance in spatial localization, pointing responses provide a valuable measure, in addition to verbal assessments. Pointing has, indeed, been used extensively in studies related to visual spatial localization (Paillard \& Beaubaton, 1978; Prablanc, Echalier, Komilis, \& Jeannerod, 1979); a few other works have used auditory pointing,

Part of these experiments was done with the help of $\mathrm{C}$. Mehauden. The authors wish to thank C. Schepens (Ligue Braille, Belgium) and all the subjects who took part in the experiments. We are indebted to J. Crémieux for his helpful criticism of the manuscript. We are also grateful to M. Meulders and to the staff of the laboratory. C. Veraart is a research associate, NFSR, Belgium.

The authors' mailing address is: Laboratory of Neurophysiology, University of Louvain, U.C.L. 5449, B-1200 Brussels, Belgium. generally with blind people (Fisher, 1964; Martinez, 1977). Meanwhile, the auditory abilities of sighted and blind persons have been compared in many studies. In some cases, superior auditory abilities-better speech discrimination (Niemeyer \& Starlinger, 1981) or more accurate spatial localization by means of auditory cues (Kellogg, 1962)-were reported for the blinds. Interpretation of these results has made use of the suggestion of some kind of sensory compensation (Hayes, 1934), even though this traditional hypothesis has been questioned (Bross \& Borenstein, 1982; Cronin, McLaren \& Campbell, 1983). Nevertheless, in other cases, delays in the development of sound-source reaching behavior has been observed with blind infants (Bower, 1977), which illustrates the major role played by vision in coordinating spatial information coming from nonvisual sensory channels (Lockman, Rieser, \& Pick, 1981). Consequently, our aim was to investigate the importance of visual experience in auditorymotor spatial integration. To do so, we restricted the testing area to the near space, where pointing responses could be more easily quantified (in direction and in distance); these motor responses were compared with verbal assessments. The amount of visual experience would be controlled by comparing the performance of blindfolded sighted, early-blind, and late-blind subjects. Finally, we tried to take into account specific strategies used by some blinds, who turn the head in order to orient the ear towards the sound source (Martinez, 1977); thus, data would be collected both with the head restrained and with the head free. The role of visual experience was also studied in tasks performed in the space of locomotion; data produced by that study will be reported elsewhere (Veraart \& Wanet, in press).

\section{METHOD}

Thirty male and female subjects, aged 21 to 54 , were included in this study. Nineteen were sighted, six were early blind, and five were late blind. 
During the experiment, the subjects sat in front of a horizontal pointing surface provided with an obvious coordinate system. A sector of $90^{\circ}$, ranging from 18 to $62 \mathrm{~cm}$, was divided into 36 tactually discriminable compartments arranged in four ranges of distance and nine ranges of direction (Figure 1). The increasing ranges of distance were labeled A, B, C, and D; the direction ranges were identified according to a clockwise code (noon in front, 8 to 11 o'clock on the left, and 1 to 4 o'clock on the right).

The sound source was a small loudspeaker, which emitted a succession of short tones ( $300 \mathrm{msec}$ each second) of fixed frequency $(800 \mathrm{~Hz})$ until the response was given. This loudspeaker, enclosed in a cylinder, was positioned on a Perspex horizontal plate set $30 \mathrm{~cm}$ above the pointing surface. Thus, the loudspeaker was at about the same height as the ears of the subjects; care was taken to point the loudspeaker toward the zero point of the coordinate system. In a typical session, this sound source was located randomly on the Perspex plate, at the vertical of the center of one of 28 compartments (distributed in the four distances and seven central directions, from $9 \mathrm{o}$ 'clock on the left to 3 o'clock on the right). After each response, the sound source was switched off and silently moved to a new location.

The position of the subject was controlled: Before each response, both of his or her forefingers lay on a tactile reference-a small piece of foam rubber at the zero point of the coordinate system. When the head was restrained, the chin and the forehead were maintained in contact with a rigid metal frame; in these conditions, the subject's face was right above the piece of foam rubber. When the head was free, the subject was asked to keep his or her face above the same reference, regardless of their other movements. In addition, the sighted subjects were blindfolded.

Two kinds of response could be given: Either the response was verbal, and the subject named the coordinates of the estimated position of the sound source (e.g., B-10 or D-2), or it was an openloop motor response, in which the subject pointed with his or her dominant forefinger, from the foam rubber, directly into the chosen compartment. The motor response was measured by recording the coordinates of the chosen compartment. For both verbal and motor tasks, the subject was allowed to select his or her response without time limits. Each subject was tested under four experimental conditions: motor responses with the head free; verbal responses,

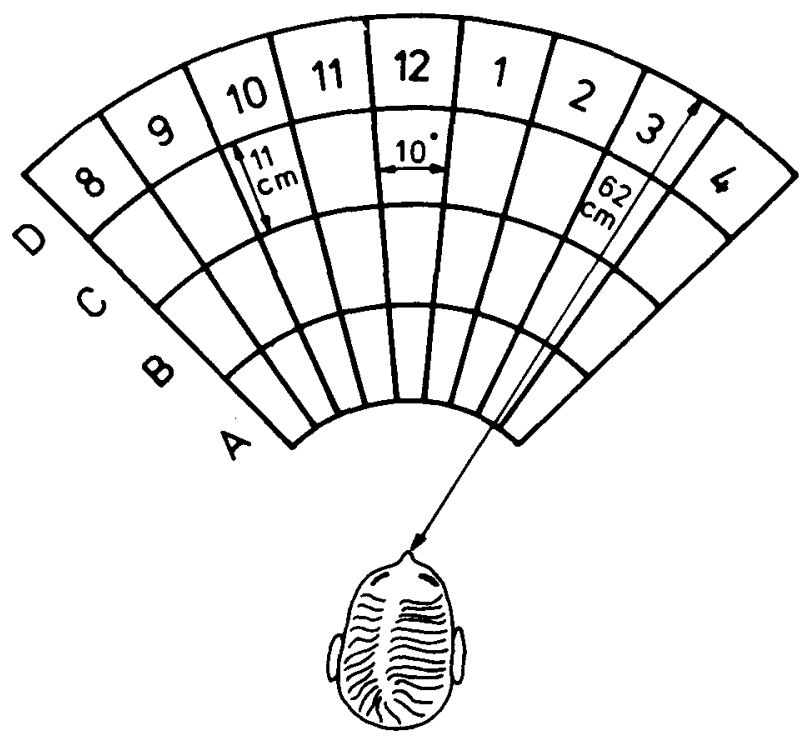

Figure 1. Outline of the experimental setup. Targets were positioned above the $\mathbf{2 8}$ middle compartments (four distances and seven directions from 9 o'clock to 3 o'clock). Pointing was recorded in all the 36 compartments shown. head free; motor responses, head restrained; and verbal responses, head restrained. For each experimental condition, the subject was asked to respond to a random presentation of the sound source (every one of the 28 possible locations being used once).

Care was taken to avoid any kind of learning, the correct response being kept unrevealed. The four experimental conditions were presented randomly.

Resulting data were processed in the following way. To each response were assigned two error values (one error value in direction and one in distance), defined as the number of compartments between the coordinates of the stimulus and those of the response. The errors in direction and in distance, related to the 28 responses for a given subject in a given experimental condition, were averaged into two mean errors.

\section{RESULTS}

In the four experimental conditions, the three groups of subjects differed in their performance as shown in Figure 2. In this figure, the results related to a given group in a given experimental condition are displayed as follows: Mean and standard deviation of the subjects' mean error values were computed for direction and distance; the horizontal axis refers to the group error in direction, and the vertical axis refers to the group error in distance. From a qualitative point of view, a rapid examination of Figure 2 shows the errors of early blind subjects to be larger than those of the two other groups; the results for sighted and late blind subjects look alike.

In order to verify these relations, we analyzed the direction and distance estimates separately by means of a onedimensional analysis of variance on the three groups of subjects (in each experimental condition). The results of this analysis are shown in Figure 3 (in the upper part of each block). It was found that there were no significant differences in the group errors for all the motor responses with the head restrained, or for all responses in direction with the head free.

When the analysis of variance was nearly significant ( $p<.10$ ), a Student $t$ test was carried out to determine which group differed from the others. In a first hypothesis, we predicted that early blinds' errors would be larger than those of sighted subjects. As shown in Figure 3 (lower part of each block), when the $F$ value was significant this prediction was substantiated in all conditions except when distance was evaluated in the verbal-response, head-restrained condition. In a second hypothesis, we predicted that early blinds would make larger errors than late blinds. This was verified only in the verbal-response, head-restrained condition for distance evaluation. Finally, we hypothesized that the performance of late blinds would not differ significantly from that of sighted subjects. This was verified in all conditions except when verbal assessments of distance were made with the head restrained; in that condition, the test showed that the late blinds' errors were unexpectedly significantly smaller than those of sighted subjects.

As far as head-free versus head-restrained performance is concerned, matched pairs comparisons were computed. Errors in distance evaluation (verbal and motor) were big- 

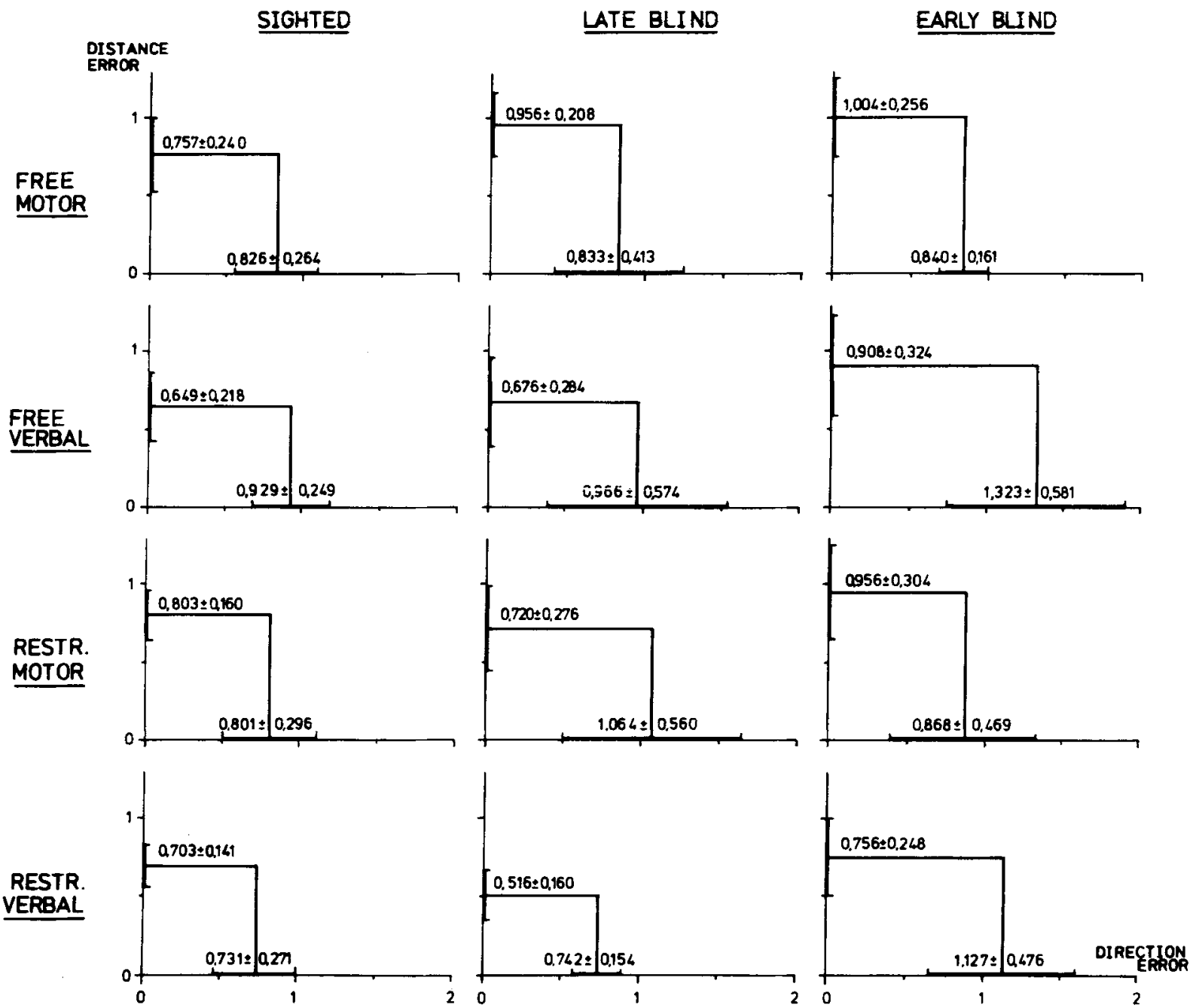

Figure 2. Motor and verbal response errors recorded in distance and in direction, when the head was free (upper two rows) or restrained (lower two rows) for the three groups of subjects. Errors are scaled in number of compartments. The mean and standard deviation are indicated graphically and numerically for each measure.

ger with the head free than with the head restrained for each blind group, but not significantly so (see Figure 2); we observed the reverse trend for the sighted subjects. Errors in direction evaluation (for the blinds as well as for the sighted) did not differ, whether the head was restrained or not, when a motor response was given; in the case of verbal responses, head-restrained errors tended to be smaller than head-free errors (significant difference for the sighted subjects).

Considering motor versus verbal performance, we hypothesized that the verbal response would be more accurate than the motor response (the latter demanding the buildup of a motor program adapted to the given auditory information). This hypothesis was supported by the results as far as distance assessments were concerned: as shown in Figure 2, errors in verbal response were always smaller than errors in motor response regardless of condition or group, although the differences were not sig- nificant. On the other hand, direction assessments tended to follow an opposite path: the errors were always smaller with motor responses than with verbal ones when the head was free, although these differences were not significant. When the head was restrained, smaller errors with motor responses for direction assessments were observed only for the early-blinds group.

\section{DISCUSSION}

Auditory spatial localization in direction and in distance was investigated as a function of visual experience, head movement, and two kinds of response.

This study was performed in the near space, using a polar coordinate system. Stimulus positioning and thus elicited response were limited to a small number of discrete values: 7 in direction and 4 in distance. The increment between two successive directions was $10^{\circ}$, in agree- 


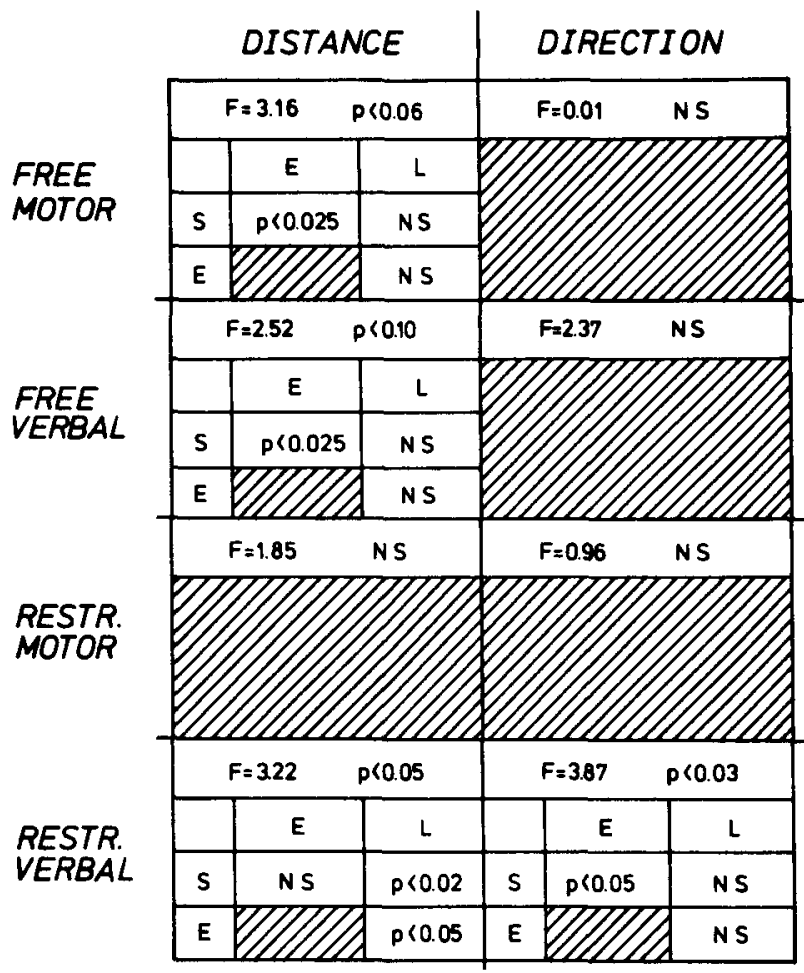

Figure 3. Significance levels in distance and in direction for statistical tests comparing the three groups of subjects among the various experimental conditions. In each of the eight main divisions, the upper portion gives the results of analysis of variance and the lower portion gives the results of Student's $t$ test when the analysis of variance was nearly significant. The shaded parts relate to not allowed comparisons. $\mathbf{E}=$ early blinds, $\mathrm{L}=$ late blinds, $\mathrm{S}=$ sighted subjects; NS $=$ not significant. In the t tests, each hypothesis always considered the early blinds' performance as lower than that of the sighted or of the late-blind subjects; in the comparisons between lateblind and sighted subjects, the hypotheses were two-tailed.

ment with data reported by Mills (1958), who, using a sound source of $750 \mathrm{~Hz}$, showed that the minimum audible angle that can be discriminated was between $1^{\circ}$ and $7^{\circ}$, depending on explored directions. Since audition is less well adapted to distance discrimination than vision, only four distance values were defined in the present experiment, the farthest one corresponding to an arm's reach, as determined by empirical trials. The 28 possibilities, defined by the distances-directions combination, were chosen randomly, each once per experiment, to constitute a 28 -item session. Four such sessions, corresponding to the four experimental conditions, seemed to constitute a maximum that would not decrease the motivation of our subjects. Statistically, our results should be reliable, especially since we dealt not with each separate item, but only with two mean values, one in direction and one in distance, in each experimental condition.

\section{Visual Experience}

According to our results, the amount of early visual experience can play a role in auditory spatial localization. Indeed, as shown by the quantitative analysis (see Fig- ure 3), early blinds, in making distance estimates, generally performed less accurately than sighted subjects and even than late blinds. Furthermore, the performance of late blinds and sighted subjects in evaluating distance appeared to be similar. For direction estimate, however, no such clear-cut conclusion could be drawn. This can be accounted for by the natural ability of the auditory system to accurately balance interaural differences and to interpret them in terms of angular direction. Results reported by Fisher (1964), in agreement with our observations, show that, in tasks of directional discrimination between two sonorous targets, early blinds' verbal responses do not differ from those of sighted subjects. Consequently, the role of early visual experience in auditory perception would apply to distance rather than direction. According to Rieser et al. (1982), one can suppose that sighted observers take advantage of a lot of sensory-motor interactions that occur as they move and that they learn the relationships between nonvisual characteristics linked to movements and their visible effects on the network of selfto-object relations; this perceptual learning would enable late blinds or blindfolded sighted subjects to update their movements in relation to features of the environment. In this view, one could accept the idea that early blinds, who have not had the opportunity to set up intersensory auditory-visual relationships, would be somewhat impaired in estimating distance, where audition remains of poor value. To conclude this discussion of the role of visual experience, two remarks about some characteristics of visual experience are necessary. First, as reflected by our results, a deficiency in early visual experience, that is, an altered development of vision, does impair spatial representation.

Second, the total amount of visual experience is not that important, inasmuch as the working duration of a normal visual system could be shorter for a given sighted subject (e.g., a 20-year-old) than for a given late blind (a 50-year-old blinded since the age of 30); obviously, a main characteristic in "visual experience" evaluation for late blinds consists in the time spent since blindness. This second parameter was not considered in the present study.

\section{Head Movements}

Head movements, when used for sound localization, are generally described in the literature (Easton, 1983, Jeffress, 1975) as improving performance. For discrimination of direction, which is based mainly on binaural cues, one can argue that active head movements create changes in the information received. According to Wallach (1940), such a modification of the binaural cues, added to the kinesthetic data obtained from the changing position of the head, gives a more accurate estimate of the sound bearing. In the same study, Wallach showed that even passive displacements of the head were useful. Possible cues to auditory distance include binaural information, loss of amplitude, frequency spectrum, and reflected sound waves (Coleman, 1963). Active head move- 
ments are not thought to provide any advantage in judging the distance of a sound (Simpson \& Stanton, 1973).

In our experimental conditions, neither direction nor distance estimates improved with the ability to make head movements, regardless of the group of subjects considered. If our results for distance are in agreement with those of other reports (Simpson \& Stanton, 1973), our failure to obtain better direction results with the head free to move needs to be discussed. We actually found that Pollack and Rose's (1967) report supported our experimental findings. Moreover, those authors suggested that head movements would give a definite advantage only for positions outside the central "cone of maximal sensitivity" of audition. Two different explanations for our results can be put forward. One could suppose that our testing positions (the farthest position on each side was $30^{\circ}$ off center) did not necessitate head movements for accurate localization. But different reasoning could explain why performance did not improve in the head-freeto-move condition. The test required both distance and direction estimates within the same response. Because perception of the sound distance is not clearly helped by head movements, the necessity to give a global response (distance and direction) could have prevented the subjects from using head movements. The present experiment does not enable us to choose definitively between these two possibilities, but some considerations about the group of early-blind people support the second hypothesis. Indeed, Martinez (1977) reported that in an experiment in which they had to point in the direction of a sound source, congenitally blind subjects used a specific strategy: they always orient the right ear towards the sound before pointing. However, we never observed this strategy in our early-blind subjects, a fact that possibly demonstrates the difference existing between a one-dimensional response (e.g., direction) and a multidimensional one (direction and distance).

\section{Motor versus Verbal Response}

An assessment of the influence of response mode (motor vs. verbal), especially in the case of early blinds, seemed to us to be important. Indeed, no study on blinds directly reports on such a comparison. One could explain this lack of data by the fact that it is generally admitted that early blinds never localize any target (sonorous or not) by pointing to it. When trying to reach a sonorous object (e.g., a musical ball), a blind child usually gropes for it rather than reaching for it directly. We bypassed this difficulty by permitting the subjects to point on (or more accurately to touch) a layout divided into tactually discriminable compartments. We explained to the subjects that the "pointing" movement had to be as direct as possible, although speed was not important; but a hesitating touch from compartment to compartment was not allowed.

With regard to the influence of response mode, our hypothesis that verbal responses would be more accurate than motor ones was supported only for distance estimates.
How can one explain that motor responses were surprisingly better than verbal ones when angular direction was involved? We propose the following explanation. Both verbal assessments and pointing refer to an auditory map or an auditory spatial representation; however, the cognitive map, reflected by verbal assessments, is obtained by means of high cognitive processes, whereas pointing reflects a motor map, which involves more automatic mechanisms. In these conditions, a better motor response in direction could reflect a more automatic performance in a task well adapted to auditory channel characteristics; similarly, a better verbal response in distance could be the result of a more computational process imposed by the inadequacy of audition in distal localization. Thus, it is not surprising that blind people try to compensate for the lack of available distance information by using artificial cues such as a long cane or more sophisticated devices. Consequently, aids for blinds that aim to substitute audition for vision should emphasize an accurate evaluation of obstacle distance, but should also respect good auditory performance in direction estimation.

\section{REFERENCES}

Bower, T. G. R. (1977). A primer of infant development. San Francisco: Freeman.

Bross, M., \& BorensteIn, M. (1982). Temporal auditory acuity in blind and sighted subjects: A signal detection analysis. Perceptual and Motor Skills, 55, 963-966.

Coleman, P. D. (1963). An analysis of cues to auditory depth perception in free space. Psychological Bulletin, 60, 302-315.

Cronin, V., Mclaren, J., \& Campbell, E. (1983). Sensory compensation in blind persons: A comparison of visual and tactual recognition. Journal of Visual Impairment and Blindness, 77, 489-499

EAston, R. D. (1983). The effect of head movements on visual and auditory dominance. Perception, 12, 63-70.

FISHER, G. H. (1964). Spatial localization by the blind. American Joumal of Psychology, 77, 2-14.

Foulke, E. (1982). Perception, cognition and the mobility of blind pedestrians. In M. Potegal (Ed.), Spatial abilities: Development and physiological foundations (pp. 55-76). New York: Academic Press.

HAYES, S. F. (1934). Sensory compensation or the vicariate of the senses. Outlook for the Blind, 28, 7-12.

JEFFRESS, L. A. (1975). Localization of sound. In W. D. Keidel \& W. D. Neff (Eds.), Handbook of sensory physiology: Vol. V/2. Auditory system (pp. 449-459). Berlin: Springer.

KellogG, W. N. (1962). Sonar system of the blind. Science, 137, 399-404.

Lockman, J. J., Rieser, J. J., \& Pick, H. L. (1981). Assessing blind travelers' knowledge of spatial layout. Journal of Visual Impairment and Blindness, 75, 321-326.

MarTinez, F, (1977). Les informations auditives permettent-elles d'établir des rapports spatiaux? Données expérimentales et cliniques chez l'aveugle congénital. Année Psychologique, 77, 179-204.

MAsterson, R. B., \& IMIG, T. J. (1984). Neural mechanisms for sound localization. Annual Review of Physiology, 46, $275-287$.

Millar, S. (1981). Crossmodal and intersensory perception and the blind. In H. L. Pick, Jr., \& R. D. Walck (Eds.), In Intersensory perception and sensory integration (pp. 281-314). New York: Plenum Press.

Mrlls, A. W. (1958). On the minimum audible angle. Journal of the Acoustical Society of America, 30, 237-246.

NiEmeyer, W., \& Starlinger, I. (1981). Do the blind hear better? Investigations on auditory processing in congenital or early acquired blindness. II. Central functions. Audiology, 20, 510-515. 
Paillard, J., \& Beaubaton, D. (1978). De la coordination visuomotrice à l'organisation de la saisie manuelle. In $\mathbf{H}$. Hecaen \& $\mathbf{M}$., Jeannerod (Eds.), Du contrôle moteur à l'organisation du geste (pp. 225-260). Paris: Masson.

Pollack, I., \& Rose, M. (1967). Effect of head movement on the localization of sounds in the equatorial plane. Perception \& Psychophysics, 2, 591-596.

Prablanc, C., Echalier, J, F., Komilis, E., \& Jeannerod, M. (1979) Optimal response of eye and hand motor systems in pointing at a visual target. I. Spatio-temporal characteristics of eye and hand movements and their relationships when varying the amount of visual information. Biological Cybernetics, 35, 113-124.

Rieser, J. J., GUth, D. A., \& HiLl, E. W. (1982). Mental processes mediating independent travel: Implications for orientation and mobility. Journal of Visual Impairment and Blindness, 76, 213-218.

Simpson, W. E., \& Stanton, L. D. (1973). Head movement does not facilitate perception of the distance of a source of sound. American Journal of Psychology, 86, 151-159.

VeraART, C., \& WANET, M. C. (in press), Sensory substitution of vision by audition. In D. H. Warren \& E. R. Strelow (Eds.), Visual spatial prostheses for the blind. The Hague: Martinus Nijhoff.

WALLACH, H. (1940). The rôle of head movements and vestibular and visual cues in sound localization. Journal of Experimental Psychology, 27, 339-368.

WARREN, D. H., \& PICK, H. L., JR. (1970). Inter-modality relations in localization in blind and sighted people. Perception \& Psychophysics, 8, 430432.

Westheimer, G. (1984). Spatial vision. Annual Review of Psychology, 35, 201-226.

(Manuscript received August 6, 1984; revision accepted for publication June 26,1985 .) 